\title{
Ecografía clínica en Medicina Interna: cuenta atrás para la universalización de su uso
}

\author{
Clinical echography in Internal Medicine: countdown for the universalisation of its use
}

\author{
Juan Torres Macho \\ Servicio de Medicina Interna-Urgencias. Hospital Universitario Infanta Cristina. Parla (Madrid). Coordinador del Grupo de trabajo de Ecografía clínica. SEMI. Departamento \\ de Medicina. Universidad Complutense de Madrid
}

José López Castro

Servicio de Medicina Interna. Hospital Comarcal de Monforte de Lemos (Lugo). Miembro del Grupo de trabajo de Ecografía clínica

En la actualidad, con la evidencia existente, casi nadie pone en duda la utilidad de la ecografía clínica en manos del internista como herramienta para potenciar su capacidad diagnóstica y terapéutica ${ }^{1}$. Tampoco hay duda de que nos encontramos ante una tecnología disruptiva que resulta sencilla de aprender a manejar y de gran utilidad. La pregunta que surge es: ¿si esto es así, por qué a día de hoy no estamos todos haciendo uso de ella?.

Toda disrupción tecnológica tiene diferentes fases e hitos que sirven como palanca de cambio para la transición desde un uso que se incrementa de forma paulatina a una expansión exponencial que llega hasta su universalización y su utilización "como si siempre hubiera estado allí". El cambio fundamental dentro de la ecografía ya ha ocurrido y es su miniaturización y portabilidad (incluso ya existen dispositivos que pueden acoplarse a "Smartphones" o "tablets"). Este hecho ha permitido llevar los ultrasonidos a la cabecera del enfermo y facilitar la repetición de la técnica las veces que haga falta (paso de la ecografía "estática" de los laboratorios de radiología o imagen cardiaca, a la evaluación ecográfica "dinámica"). Esta valoración ecográfica evolutiva está abriendo múltiples posibilidades en el manejo de distintas patologías (monitorización hemodinámica no invasiva, seguimiento del paciente con insuficiencia cardiaca....etc ${ }^{2}$.

Todavía existen profesionales sanitarios (incluidos algunos internistas y médicos de familia) que son escépticos en relación a la utilidad de la ecografía clínica en manos del generalista. Este rechazo, aunque es un denominador común cuando surgen tecnologías disruptivas, no tiene ninguna base más allá de inquietudes relacionados con un posible intrusismo o la resistencia al cambio y estos argumentos están poco justificados. Cualquier profesional que entienda el concepto de ecografía clínica sabe que esta herramienta no es equivalente ni tiene el mismo fin que un estudio ecográfico completo realizado en un laboratorio de ecografía o imagen cardiaca, además su uso generalizado es indudablemente beneficioso para los pacientes (objetivo principal de nuestra actividad) y de hecho ya está mejorando el pronóstico de muchos pacientes ${ }^{3}$. También es cierto que resulta imprescindible asegurar la formación y el uso adecuado de la técnica, conocer su utilidad y alcance, los posibles sesgos y establecer un itinerario formativo normalizado y ésto es una responsabilidad fundamental que tienen las diferentes sociedades científicas implicadas que deberán actuar de forma coordinada en los próximos años.

Sin duda alguna, la ecografía clínica nos puede hacer mejores profesionales si se utiliza de forma adecuada. Lógicamente cuanto mayor es el conocimiento clínico y la pericia previa en la elaboración de la anamnesis y la exploración física, mayor rendimiento se puede obtener de la herramienta y no nos debemos llevar a engaños: un buen médico con un ecógrafo puede ser mejor médico, pero la ecografía no suple nuestras posibles deficiencias previas.

Como ejemplo de la indudable utilidad de esta herramienta, en este número Remesal et al, realizan una revisión actualizada de la utilidad de la ecografía clínica en el manejo del paciente con disnea de origen incierto. La disnea es uno de los paradigmas del diagnóstico diferencial para el generalista. Tal y como se comenta en el artículo, la ecografía clínica permite orientar el diagnóstico etiológico y el tratamiento en estos pacientes con una fiabilidad y rapidez superior a otras pruebas 


\section{"un buen médico con un ecógrafo puede ser mejor médico, pero la ecografía no suple nuestras posibles deficiencias previas"}

radiológicas 0 analíticas disponibles. Además actualmente existen datos ecográficos con una importante capacidad pronóstica que pueden ser incorporados en el seguimiento evolutivo de algunas patologías muy prevalentes ${ }^{4,5}$. El trabajo concluye con un algoritmo sencillo y original para la valoración rápida de la disnea a pie de cama. Estos estudios contribuyen a sistematizar el vasto cuerpo de conocimiento de la ecografía clínica y conforman la mejor evidencia de que esta técnica aporta al clínico práctico mucho más que una mera aproximación o curiosidad anatómica, modificando frecuentemente el manejo de la patología que presenta el enfermo.

Por lo comentado previamente, parece que todavía nos falta un pequeño empujón para la explosión definitiva. Hay dos pasos necesarios que parecen evidentes: el primero es el abaratamiento de los equipos portátiles, que es una cuestión inevitable y que no parece que vaya a demorarse mucho en el tiempo y el otro es la inclusión de la ecografía clínica en el programa formativo de las distintas especialidades, exigiendo en particular a los médicos residentes de Medicina Interna la adquisición de habilidades y destrezas en ecografía clínica básica.

Podríamos añadir un tercer factor clave que es la introducción de la ecografía clínica en la enseñanza pregrado. Existen numerosas experiencias a nivel internacional y nacional que muestran la utilidad de la ecografía en la enseñanza de conceptos anatómicos, fisiológicos y fisiopatológicos con resultados notables. En este sentido uno de los factores limitantes es la potencial necesidad de profesorado con formación en ecografía, aunque existen experiencias recientes que han propuesto soluciones basadas en la participación y colaboración de los alumnos ${ }^{6}$.
Una vez que ocurran estos hechos ya será cuestión de muy poco tiempo que resulte impensable realizar una auscultación cardiaca que no esté complementada por una ecografía cardiaca básica, igual que ahora parecería impensable no realizar y saber interpretar una radiografía de tórax ante una auscultación patológica. Lo que cada uno tendrá que decidir de forma personal es cuando va a empezar a formarse e incorporar la ecografía clínica en su quehacer diario. Desde el Grupo de trabajo de Ecografía clínica de la Sociedad Española de Medicina Interna lo tenemos claro: hoy mejor que mañana. Es probable que todo internista "joven" tenga que aprender a utilizar la ecografía clínica igual que ahora está obligado a saber interpretar un electrocardiograma, ya que será una habilidad que resultará imprescindible para el óptimo desempeño de su práctica habitual.

Concretamente en Galicia y desde SOGAMI, debemos vencer la inercia al cambio y ser motores de la implantación de una técnica económica que bien utilizada, redunda en un importante beneficio para el paciente con la consecuente satisfacción para el profesional.

\section{Bibliografía}

1. Beltrán LM, García-Casasola G. La ecografía en manos del internista. ¿El estetoscopio del siglo XXI?. Rev Clin Esp. 2014; 214(3): 155-60.

2. Levitov A, Frankel HL, Blaivas M, Kirkpatrick AW, Su E, Evans D, Summerfield DT, Slonim A, Breitkreutz R, Price S, McLaughlin M, Marik PE, Elbarbary M. Guidelines for the Appropriate Use of Bedside General and Cardiac Ultrasonography in the Evaluation of Critically III Patients-Part II: Cardiac Ultrasonography. Crit Care Med. 2016; 44:1206-27.

3. Torres Macho J, García de la Torre P, de Castro García M, García de Casasola G. Ecocardiografía clínica básica en urgencias: ¿podemos mejorar el pronóstico de algunos pacientes?. Emergencias 2015;27;204-210.

4. Picano E, Pellikka PA. Eur Heart J. 2016. Epub ahead of print.

5. Cubo-Romano P, Torres-Macho J, Soni NJ, Reyes LF, Rodríguez-Almodóvar A, Fernández-Alonso JM, González-Davia R, Casas-Rojo JM, Restrepo MI, de Casasola GG. Admission inferior vena cava measurements are associated with mortality after hospitalization for acute decompensated heart failure. J Hosp Med. 2016 Jun 6. Epub ahead of print.

6. García de Casasola Sánchez G, González Peinado D, Sánchez Gollarte A, Muñoz Aceituno E, Peña Vázquez I, Torres Macho J. Enseñanza de la ecografía clínica en el pregrado: Ios estudiantes como mentores. Rev Clin Esp. 2015;215:211-6. 